\title{
Deformations and Liquefaction of Granular Soils in Plane Strain Conditions
}

\author{
Andrzej Sawicki, Justyna Sławińska \\ Institute of Hydro-Engineering Polish Academy of Sciences, ul. Kościerska 7, 80-382 Gdańsk, Poland, \\ e-mails: as@ibwpan.gda.pl, stynaju@ibwpan.gda.pl
}

(Received September 14, 2011; revised March 07, 2012 )

\begin{abstract}
An incremental model is proposed to describe pre-failure behaviour of granular soils in plane strain conditions. The model has been derived from an approach applied in the analysis of triaxial tests. We have applied the methods of tensor algebra to transfer our triaxial results to a $2 \mathrm{D}$ case. The model describes the pre-failure deformations of fully drained sands and phenomena associated with undrained response such as static liquefaction. This model has a simple structure in the form of incremental equations. For some simple loading paths, these equations can be integrated analytically. For more complex loading paths a simple numerical algorithms will be sufficient. The model takes into account an initial anisotropy of soil which is ignored in most models. It also takes into account the initial state of soil, defined as rather contractive or dilative, in addition to classical geotechnical division into loose and dense sands. The proposed constitutive equations allow the study of pre-failure instabilities of sand, and some examples are presented. We have also derived analytically the equation for an instability line.
\end{abstract}

Key words: granular soils, deformations, liquefaction, stability, plane strain conditions

\section{Introduction}

The plane strain behaviour of granular soils, both dry/fully drained and undrained, is analysed using a simple incremental model. The model has been developed specially for plane strain conditions, as they are often assumed in various analyses of soil mechanics and foundation engineering problems. It was assumed that the intermediate principal stress does not influence the soil behaviour, which leads to a rather simple structure of constitutive equations that may be useful in numerical calculations. Note that we have followed a basic idea of the Coulomb-Mohr yield condition, where the intermediate principal stress is not taken into account.

Our 2D model has been formulated on the basis of incremental equations proposed for triaxial conditions by Sawicki and Świdziński (2010a, b), which have already been re-formulated for 3D and 2D conditions, using the methods of tensor algebra, see Sawicki (2008). In this paper, some basic results of that approach will be summarized 
to make our presentation self-consistent. It is shown how the model can be calibrated on the basis of experimental data obtained from the triaxial tests, which are tool in each geotechnical laboratory. This enables each researcher to check our approach and validate our model using his own data.

Unfortunately plane strain apparatuses are quite rare in geotechnical laboratories; therefore, we must to rely mainly on data from triaxial tests. Our paper has been inspired partly by the paper of Chu and Wanatowski (2009), who have constructed a plane strain apparatus and performed a series of various tests. It seems that such experiments may introduce some new prospects to geomechanics and may indicate some new research directions. Please note that our model has been calibrated in different conditions, and for different granular soil.

A general problem in geomechanics is that there exist tens of various models, but none of them is sufficiently good to describe the pre-failure behaviour of granular soils. The basic paradigm in soil mechanics is rooten in elasto-plastic approach, which has become almost a standard in geomechanical investigations. The other problem is that these various approaches have not been validated sufficiently well on the basis of experimental data, as shown by some workshops, see Saada and Bianchini (1987). The experiments used to validate some of these models were performed in different laboratories, using different methods and materials. Our approach, see Sawicki and Świdziński (2010a, b), is based on a sound empirical background of hundreds of experiments, performed in the same laboratory, on the same soil and using the same methods. It should be added that some researchers have noticed certain shortcomings of the elasto-plastic approach to geomechanical problems, and have proposed such alternatives as hypoplasticity (Kolymbas 2000a, b) or endochronic approaches (Valanis and Read 1982, Bazant and Krizek 1976).

The model presented in this paper takes into account such features as the initial state of soil, defined either as contractive or dilative; initial anisotropy; and pre-failure instabilities. In the case of fully saturated soil, in undrained conditions, the model describes such phenomena as the pore-pressure generation and subsequent liquefaction.

\section{Some Remarks about Methodology}

In the first step of our investigations, we have compared our extensive experimental data with some predictions of commonly accepted models (elasto-plastic and hypoplastic), and we have discovered that those models lead to wrong predictions, Głębowicz (2006), Sawicki (2003). This is not a surprise, as numerous workshops have led to similar conclusions, cf. Saada and Bianchini (1987). Therefore, we have decided to elaborate a model that is based on rough experimental data. We have elaborated, in detail, a huge set of experiments, performed in triaxial apparatus, and proposed a simple incremental model describing the pre-failure behaviour of granular soils. This model describes very well the soil behaviour, as it is rooted firmly in an empirical background. We would like to present the results of our investigations in 
form useful to geotechnical engineers. Please note that it can be difficult to follow most of the various soil models, not to mention their practical applications. Our approach is different as it can be applied by an average geotechnical engineer, without the need to spend many hours studying our contribution to understand how to apply it in practice.

The first step is to understand the triaxial behaviour of sand. There appear such notions as the instability line, the initial state defined as contractive or dilative, anisotropy etc. We can hardly find such definitions in basic geotechnical textbooks. Our experimental results have confirmed many previously described phenomena and have allowed the discovery of some new features of the behaviour of granular soils. Our incremental equations (19) and (20) have been checked for various conditions, and it was proved that they lead to reasonable predictions, see Sawicki and Świdziński $(2010 \mathrm{a}, \mathrm{b})$. We have decided to extend the results obtained to the more general cases of 3D and 2D (see Sawicki 2008).

The 2D approach, presented in this paper, is mainly of practical character. We extend our results to problems which are important in practice, as most geotechnical problems are analysed in $2 \mathrm{D}$ conditions. The problem lays in how to generalize the triaxial conditions onto different configurations such as, for example, 2D conditions. It has been found that the methods of tensor algebra are most appropriate in this case. Such a method was also applied by Mróz et al (2003) to extend their elasto-plastic model of Superior sand to 3D and 2D conditions. As the result of these investigations, we present respective constitutive equations and study their behaviour for various loading conditions. As was already mentioned, we have calibrated our 2D model using the results from triaxial investigations, as we have no access to the plane strain apparatus.

\section{Incremental Model}

Consider the plane strain conditions, where the vertical axis $x_{1}$ denotes a privileged direction with respect to initial anisotropy, and the horizontal axis is denoted as $x_{3}$. The strain and effective stress tensors are the following in this case:

$$
\begin{aligned}
\boldsymbol{\varepsilon} & =\left[\begin{array}{ll}
\varepsilon_{11} & \varepsilon_{13} \\
\varepsilon_{13} & \varepsilon_{33}
\end{array}\right], \\
\boldsymbol{\sigma}^{\prime} & =\left[\begin{array}{ll}
\sigma_{11}^{\prime} & \sigma_{13}^{\prime} \\
\sigma_{13}^{\prime} & \sigma_{33}^{\prime}
\end{array}\right] .
\end{aligned}
$$

Their deviators are:

$$
\varepsilon^{d e v}=\frac{1}{2}\left[\begin{array}{ll}
\varepsilon & \gamma \\
\gamma & -\varepsilon
\end{array}\right]
$$




$$
\sigma^{\prime d e v}=\frac{1}{2}\left[\begin{array}{ll}
\sigma & 2 \tau \\
2 \tau & -\sigma
\end{array}\right],
$$

where: $\varepsilon=\varepsilon_{11}-\varepsilon_{33}, \gamma=2 \varepsilon_{13}, \sigma=\sigma_{11}^{\prime}-\sigma_{33}^{\prime}, \tau=\sigma_{13}^{\prime}$.

Similar relations are valid for the stress and strain increments $d \sigma^{\prime}, d \varepsilon$. The proposed incremental equations, describing the pre-failure deformations of soil, are of the following form:

$$
\begin{gathered}
d \varepsilon_{v}=A d p^{\prime}+B d J_{2}, \\
d \varepsilon^{d e v}=\mathbf{C} d p^{\prime}+D d \boldsymbol{\sigma}^{\prime d e v},
\end{gathered}
$$

where $d \varepsilon_{v}=d \varepsilon_{11}+d \varepsilon_{33}$ is the increment of volumetric strain $) / 2, d p^{\prime}=\left(d \sigma_{11}+\right.$ $\left.d \sigma_{33} / 2\right)$ is the increment of the mean effective stress, $A, B$ and $D$ are constitutive functions, $d J_{2}=(\sigma d \sigma) / 2+2 \tau d \tau$ is the increment of the second invariant of stress deviator as $J_{2}=\sigma^{2} / 4+\tau^{2}$. Tensor $\mathbf{C}$ describes an initial anisotropy of soil (transverse isotropy with the vertical privileged direction), and is defined as

$$
\mathbf{C}=C \mathbf{S}^{d e v},
$$

where $C$ is a function and

$$
\mathbf{S}^{d e v}=\frac{1}{2}\left[\begin{array}{ll}
1 & 0 \\
0 & -1
\end{array}\right]
$$

is the deviator of structural tensor

$$
\mathbf{S}=\left[\begin{array}{ll}
1 & 0 \\
0 & 0
\end{array}\right]
$$

Note that Eq. (5) has a scalar form whilst Eq. (6) is of tensorial character. In the case of initially isotropic soil, $C=0$. The function $B$ describes the effect of dilation, which is a characteristic feature of granular materials. For example, for an ideal elastic and isotropic body, we have $B=0$ and $C=0$, and functions $A$ and $D$ are just numbers that depend on the elastic moduli. Eq. (6) can be re-written in the form of two scalar equations:

$$
\begin{gathered}
d \varepsilon=C d p^{\prime}+D d \sigma, \\
d \gamma=2 D d \tau .
\end{gathered}
$$

Therefore, the model proposed is described by three constitutive equations (5), (10) and (11).

It should also be noted that the assumed definition of the mean effective stress in $2 \mathrm{D}$ conditions can be derived from a general formula $p^{\prime}=\left(\sigma_{11}^{\prime}+\sigma_{22}^{\prime}+\sigma_{33}^{\prime}\right) / 3$ when we assume $\sigma_{22}^{\prime}=\left(\sigma_{11}^{\prime}+\sigma_{33}^{\prime}\right) / 2$. This means that the intermediate normal stress is just an average from the extreme normal stresses. 


\section{Loading and Unloading}

In mechanics of inelastic materials it is important to distinguish the loading and unloading. In classical elasto-plasticity, during the loading both the plastic and elastic strains develop, whilst during unloading only the elastic strains are recovered. In order to distinguish between loading and unloading, the yield surfaces are introduced. If the stress increment is directed outwards from the current yield surfaces or is tangent to that surface, the process of loading takes place. If the stress increment is directed inwards from this surface, we deal with unloading. However, the problem is that the same stress increment can be identified with loading according to one model, and with unloading according to another model. Such a dilemma should be settled on the basis of convincing experimental data, but unfortunately such publications are rare in geotechnical literature.

We have introduced an alternative definition of loading and unloading which is independent of any yield surface, and has an objective character. This definition is based on classical decomposition of the stress tensor onto the spherical and deviatoric parts. The spherical part of the stress tensor, i.e. the mean effective stress plays a constructive role as the soil skeleton becomes stronger when $p^{\prime}$ increases. In the case of $p^{\prime}=0$ the soil skeleton does not exist anymore and we have a mere assembly of grains. The role of the stress deviator is "destructive" as the soil skeleton becomes weaker when it increases, and in the extreme case a failure takes place. Therefore, we introduce the spherical loading/unloading and the deviatoric loading/unloading. They are defined as follows:

$$
d p^{\prime}>0-\text { spherical loading, } \quad d p^{\prime}<0-\text { spherical unloading. }
$$

Let us introduce the following non-dimensional stress variable;

$$
\eta=\frac{\sqrt{3 J_{2}}}{p^{\prime}}
$$

which is a generalization of well known variable $\eta=q / p^{\prime}$ applied in the analysis of triaxial tests, where $q$ denotes respective deviatoric stress. The increment of this variable is the following:

$$
d \eta=\frac{\sqrt{3}}{p^{\prime}}\left(\frac{1}{2 \sqrt{J_{2}}} d J_{2}-\frac{\sqrt{J_{2}}}{p^{\prime}} d p^{\prime}\right) .
$$

The respective definition is the following:

$$
d \eta>0-\text { deviatoric loading }, \quad \mathrm{d} \eta<0-\text { deviatoric unloading. }
$$

In this paper, the soil mechanics sign convention is used, where the plus sign denotes compression. The functions $A, B, C$ and $D$, appearing in the incremental equations (5), (10) and (11) are different for loading and unloading. 


\section{Instability Surface}

During triaxial investigations of granular soils, it was discovered that there exists, in the effective stress space, the so-called instability line; see Sladen et al (1985), Lade et al (1987), Lade (1992). Further investigations, e.g. Sawicki and Świdziński (2010a, b), have shown that this line can even be derived analytically from incremental constitutive equations, formulated for the triaxial configuration. In the case of dry/fully drained sand, at initially dilative state, this line corresponds to the change from compaction to dilation due to shearing. In the case of initially contractive saturated sand, tested in undrained conditions, the instability line corresponds to the maximum shear stress that can be supported by the soil skeleton. After reaching this line, the phenomenon of static liquefaction takes place. For the triaxial configuration, the instability line is defined by the following equation:

$$
q=\Psi p^{\prime},
$$

where $\Psi$ is a certain number. In the case of model "Skarpa" sand, used in investigations by Sawicki and Świdziński (2010a, b), this coefficient is in the range 0.9-1. Eq. (16) is equivalent to the following relation, expressed in terms of the stress invariants:

$$
J_{2}=\frac{1}{3} \Psi^{2}\left(p^{\prime}\right)^{2} .
$$

For the case considered in this paper, Eq. (17) takes the following form:

$$
\frac{1}{4} \sigma^{2}+\tau^{2}=\frac{1}{3} \Psi^{2}\left(p^{\prime}\right)^{2}
$$

which represents a cone in the stress space.

Let us examine some experimental results presented in Chu and Wanatowski (2009). It follows from their Fig. 7a, on page 116, that the instability line is given by the following formula: $\eta_{I L}=q / p^{\prime}=0.89$ where $q=\sqrt{3 J_{2}}$ and $p^{\prime}$ is defined for a general 3D case. Note that their value is only slightly different from a slope of instability line defined for the "Skarpa" sand (recall $\Psi=\eta_{I L}=0.91$ ).

\section{Calibration of the Model}

\subsection{Indirect Calibration in Triaxial Apparatus}

The most direct method of calibration of the model would be determination of the functions $A, B, C$ and $D$, appearing in Eqs. (5), (10) and (11), directly from the tests performed in the plane strain apparatus. The authors of the present contribution have not had such a possibility, and therefore Sawicki (2008) has proposed a method of determination of these functions from the triaxial tests. The incremental equations, formulated for the triaxial configuration, are of the following form:

$$
d \varepsilon_{v}=M d p^{\prime}+N d q,
$$




$$
d \varepsilon_{q}=P d p^{\prime}+Q d q
$$

where $M, N, P$ and $Q$ are functions determined from a set of experiments, performed in the triaxial apparatus. Recall that they are different for loading and unloading. Tables 1 and 2 show these functions, after Sawicki and Świdziński (2010a). Only function $N$ for initially dilative sand is presented in simpler form than before. There appear some coefficients, whose values will be presented in subsequent sections. The other symbols, appearing in Eqs. (19) and (20), have the following meaning: $q=\sigma_{1}^{\prime}-\sigma_{3}^{\prime}$ $=$ stress deviator in triaxial configuration (difference between the vertical and horizontal effective stresses); $\varepsilon_{v}=\varepsilon_{1}+2 \varepsilon_{3}=$ volumetric strain in triaxial configuration; $\varepsilon_{q}=2\left(\varepsilon_{1}-\varepsilon_{3}\right) / 3=$ respective deviatoric strain. The subscripts 1 and 3 denote the vertical and horizontal directions respectively.

Table 1. The functions $M, N, P, Q$ for loading

\begin{tabular}{|c|c|c|}
\hline Function & contractive & dilative \\
\hline M & & $\frac{A_{v}}{2 \sqrt{p^{\prime}}}$ \\
\hline$N$ & $\frac{4 c_{1} \eta^{3}}{\sqrt{p^{\prime}}}$ & $\begin{array}{c}\frac{1}{\sqrt{p^{\prime}}}\left(2 a_{1} \eta+a_{2}\right) ; \text { for } 0 \leq \eta \leq \eta^{\prime}, \\
\frac{1}{\sqrt{p^{\prime}}}\left[a_{3} a_{5} \eta+a_{3}+a_{4} a_{5}\right] \exp \left(a_{5} \eta\right) \text { for } \eta^{\prime} \leq \eta \leq \eta^{\prime \prime}\end{array}$ \\
\hline$P$ & & $\frac{A_{q}}{2 \sqrt{p^{\prime}}}$ \\
\hline$Q$ & & $\frac{b_{1} b_{2}}{\sqrt{p^{\prime}}} \exp \left(b_{2} \eta\right)$ \\
\hline
\end{tabular}

Table 2. The functions $M, N, P, Q$ for unloading

\begin{tabular}{|c|c|}
\hline Function & \\
\hline $\mathrm{M}$ & $\frac{A_{v}^{u}}{2 \sqrt{p^{\prime}}}$ \\
\hline $\mathrm{N}$ & $\frac{a_{v}}{\sqrt{p^{\prime}}}$ \\
\hline$P$ & $\frac{A_{q}^{u}}{2 \sqrt{p^{\prime}}}$ \\
\hline$Q$ & $\frac{b_{q}}{\sqrt{p^{\prime}}}$ \\
\hline
\end{tabular}

The above relations and functions have been generalized using the methods of tensor algebra, see Sawicki (2008). The main result of those investigations was a derivation of relationships between the functions $A, B, C, D$, appearing in the plane strain 
model and the functions $M, N, P, Q$ derived for the triaxial conditions. Respective relationships are as follows:

$$
A=M ; \quad B=\frac{N \sqrt{3}}{2 \sqrt{J_{2}}} ; \quad C=\frac{3 P}{2} ; \quad D=\frac{3 Q}{2} .
$$

\subsection{Procedure for Calibration in Plane Strain Apparatus}

In the present section, a procedure for calibration of the model in plane strain apparatus will be described. Eqs. (5) and (10) can be re-written in the following form:

$$
\begin{aligned}
& d \varepsilon_{v}=A d p^{\prime}+\frac{2}{3} B q d q, \\
& d \varepsilon=C d p^{\prime}+\frac{2}{\sqrt{3}} D d q,
\end{aligned}
$$

where

$$
q=\frac{\sqrt{3} \sigma}{2}=\frac{\sqrt{3}\left(\sigma_{1}-\sigma_{3}\right)}{2} .
$$

In this and subsequent sections, in place of the more general notation introduced in Section 3, the principal stresses and strains will be used, as in the plane strain tests there is: $\tau=0$ and $\gamma=0$. This means that $\sigma_{11} \equiv \sigma_{1} ; \sigma_{33} \equiv \sigma_{3} ; \varepsilon_{11} \equiv \varepsilon_{1} ; \varepsilon_{33} \equiv \varepsilon_{3}$, see Fig. 1.

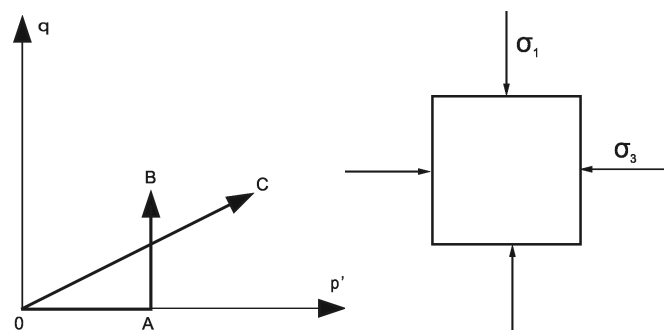

Fig. 1. Stress paths in the stress space

The experiments should be performed on dry or saturated, but fully drained samples, for different initial states, defined here in a classical manner, i.e. loose and dense. The first set of experiments deals with application of the mean stress $p^{\prime}$ (path 0A in Fig. 1). In this case $d q=0$ and Eqs. (22) and (23) reduce to a simple form in which only functions $A$ and $C$ will appear. These functions can easily be determined from experimental data, as we can construct respective curves:

$$
\varepsilon_{v}=\varepsilon_{v}\left(p^{\prime}\right), \varepsilon=\varepsilon\left(p^{\prime}\right) .
$$

These curves can be approximated by analytical formulae, and such formulae should then be differentiated with respect to $p^{\prime}$. For example, in the case of triaxial tests, we 
have found that the volumetric strains can be described by the following function: $\varepsilon_{v}=A_{v} \sqrt{p^{\prime}}$, where $A_{v}$ are numbers, obviously different for initially loose or dense sands. Then, the above equation was differentiated, and the following incremental equation has been obtained: $d \varepsilon_{v}=\left(A_{v} / 2 \sqrt{p^{\prime}}\right) d p^{\prime}=M d p^{\prime}$. Each experiment should be performed for spherical loading (path $0 \mathrm{~A}$ ) and unloading (path $\mathrm{A} 0$ ).

The second set of experiments deals with pure shearing of samples at constant mean stress $p^{\prime}=p_{A}^{\prime}=$ const (path $\mathrm{AB}$ for deviatoric loading and path BA for deviatoric unloading, see Fig. 1). Experiments should be performed for different values of $p_{A}^{\prime}=$ const. In this case $d p^{\prime}=0$ and Eqs. (22) and (23) also reduce to a simpler form, in which only functions $B$ and $D$ will appear. Respective experimental curves can then be plotted:

$$
\varepsilon_{v}=\varepsilon_{v}\left(q, p_{A}^{\prime}\right) ; \varepsilon=\varepsilon\left(q, p_{A}^{\prime}\right)
$$

These curves should be determined separately for initially contractive and dilative soils. It is an important distinction as the behaviour of initially contractive and dilative soils during shearing is different. Initially contractive soils compact during shearing. Initially dilative soils compact at the beginning of shearing and then dilate. In order to define whether a sample is contractive or dilative, one should plot a point $e, p^{\prime}$ corresponding to initial void ratio $e$ and applied mean effective stress in the space $e-\log p^{\prime}$, and find whether that point lies above or below the Steady State Line (SSL). Obviously, SSL should be determined first, using known methods, see Sawicki and Świdziński (2010a).

An important issue is analytical approximation of experimental curves (25). Sawicki and Świdziński (2010a) have found an efficient method of dealing with such data. By introducing new variables, such curves can be plotted in the form of common or "universal" curves. This means that curves corresponding to various values of $p_{A}^{\prime}$ can be plotted as a single curve. Having determined functions $A, B, C$ and $D$, one can check predictions of the model for loading paths different from those used for calibration, for example, path $0 \mathrm{C}$ in Fig. 1. The model also applies in the case of undrained behaviour, see Section 9.

\section{Determination of Strains for Simple Loading Paths}

In this Section, it will be shown how to integrate incremental equations (22) and (23) for some simple loading paths, such as those shown in Fig. 1. It is assumed that these equations have been calibrated using the method described in Section 6.1, which means that the results obtained correspond to "Skarpa" sand. We do not have access to experimental data from the plane strain apparatus corresponding to that sand, so we shall present predictions which could eventually be checked experimentally. 


\subsection{Characteristic Objects}

The stress paths shown in Fig. 1 are presented in the stress space $q, p^{\prime}$. A similar analysis could be presented in the space of principal stresses $\sigma_{1}, \sigma_{3}$ as well, but it is a matter of convenience. In each space, there are two important characteristic objects which can be presented as lines. The first one corresponds to the Coulomb-Mohr yield condition. In the case considered, this is given by the following equation:

$$
q=\sqrt{3} p^{\prime} \sin \varphi
$$

where $\varphi$ denotes the angle of internal friction.

For the "Skarpa" sand $\left(\varphi=34^{\circ}\right)$ we obtain $q=0.97 p^{\prime}$. The sand analyzed by Chu and Wanatowski (2009) is characterized by $\varphi \cong 48^{\circ}$. It gives $q=1.29 p^{\prime}$. It follows from their Fig. 7a that $q=1.16 p^{\prime}$, which means that $\varphi=42^{\circ}$. This value corresponds to the critical state (CSL), which is often identified with failure, i.e. the Coulomb-Mohr yield condition. However, from their Fig. 4a it follows that the angle of internal friction at failure is $62^{\circ}$ which is an unusual result.

The second important object is the instability line. We have discovered some interesting properties of this object. The first one is the behaviour of dry or saturated sand, but in drained conditions during shearing. In the case of initially dilative soil, such a sand initially compacts, but after reaching the instability line the process of dilation takes place. In undrained conditions, static liquefaction takes place, but in the case of initially contractive soil. As discussed before, the instability line, defined by Chu and Wanatowski (2009), in their Fig. 7a is almost the same as that for the "Skarpa" sand. The instability line is given by the following expression in the case considered:

$$
q=\Psi p^{\prime} .
$$

\subsection{Spherical Loading}

Spherical loading takes place along path 0A in Fig. 1 and Eqs. (22) and (23) reduce to the following form:

$$
\begin{gathered}
d \varepsilon_{v}=A d p^{\prime}=M d p^{\prime}=\frac{A_{v}}{2 \sqrt{p^{\prime}}} d p^{\prime}, \\
d \varepsilon=C d p^{\prime}=\frac{3 A_{q}}{4 \sqrt{p^{\prime}}} d p^{\prime} .
\end{gathered}
$$

Integration of these equations with zero initial conditions leads to simple formulae:

$$
\begin{aligned}
& \varepsilon_{V}=A_{v} \sqrt{p^{\prime}}, \\
& \varepsilon=\frac{3}{2} A_{q} \sqrt{p^{\prime}} .
\end{aligned}
$$


The values of respective coefficients have been determined for stress unit $10^{5} \mathrm{~N} / \mathrm{m}^{2}$ and strain unit $10^{-3}$. For initially loose "Skarpa" sand $\left(I_{D}=0.016-0.445\right)$ their average values are the following: $A_{v}=6, A_{q}=-0.95$. For initially dense sand $\left(I_{D}=\right.$ $0.71-0.86)$ their average values are $A_{v}=3.47, A_{q}=-0.53$. In order to become familiar with the introduced units let us consider the following example. Calculate the volumetric strain caused by the mean stress $p^{\prime}=200 \mathrm{kPa}=2 \times 10^{5} \mathrm{~N} / \mathrm{m}^{2}$ for initially loose sand. Eq. (30) gives: $\varepsilon_{v}=6 \sqrt{2}=8.49$ in respective strain unit. This means that the volumetric strain is $8.49 \times 10^{-3}=0.00849$. Such units are most convenient in numerical calculations as we manipulate with numbers of similar orders of magnitude.

Note that Eq. (31) describes the deviatoric strains caused by the mean stress. In the case of initially isotropic soil such strains should be equal to zero. Non-zero deviatoric strain means that the soil is initially anisotropic. This is a cross-anisotropy caused by gravity. Most of the models of soils do not take into account this feature, and they assume that the soil is initially isotropic. Fig. 2 illustrates the volumetric and deviatoric strains that develop during spherical loading for the average values of material parameters.

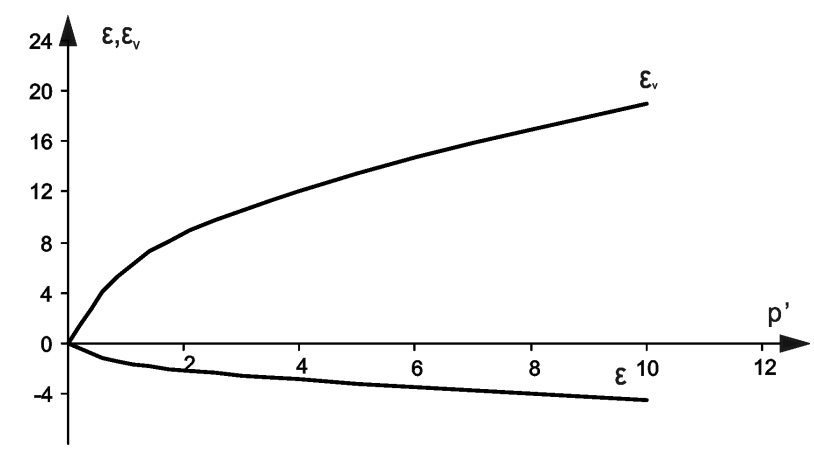

Fig. 2. Volumetric and deviatoric strains that develop during spherical loading

\subsection{Deviatoric Loading}

Deviatoric loading takes place along path AB in Fig. 1 and Eqs. (22) and (23) are of the following form:

$$
\begin{aligned}
& d \varepsilon_{v}=\frac{2}{3} B q d q=N d q, \\
& d \varepsilon=\frac{2 D}{\sqrt{3}} d q=\sqrt{3} Q d q .
\end{aligned}
$$

Recall that function $N$ has a different shape for initially contractive and dilative sand. Therefore, we should consider these two cases separately. 


\section{Initially contractive sand}

In this case, Eqs. (32) and (33) take the following form:

$$
\begin{gathered}
d \varepsilon_{v}=\frac{4 c_{1} q^{3}}{\left(p^{\prime}\right)^{7 / 2}} d q, \\
d \varepsilon=\frac{\sqrt{3} b_{1} b_{2}}{\sqrt{p^{\prime}}} \exp \left(\frac{b_{2}}{p^{\prime}} q\right) d q .
\end{gathered}
$$

Note that we have chosen $\mathrm{q}$ as an independent variable, but we could just as well use another independent variable, such as $\eta=q / p^{\prime}$. Integration of the above equations is simple, and leads to the following formulae:

$$
\begin{gathered}
\varepsilon_{v}=\varepsilon_{v}\left(p_{A}^{\prime}\right)+\frac{c_{1} q^{4}}{\left(p^{\prime}\right)^{7 / 2}}, \\
\varepsilon=\varepsilon\left(p_{A}^{\prime}\right)+\sqrt{3} b_{1} \sqrt{p^{\prime}}\left[\exp \left(\frac{b_{2}}{p^{\prime}} q\right)-1\right] .
\end{gathered}
$$

The first members on the RHS of Eqs. (36) and (37) denote the strains that have developed during spherical loading, preceding the pure shearing considered in this Section.

The coefficients appearing in the above equations have been determined experimentally for "Skarpa" sand. Their values are as follows: $c_{1}=2.97 ; b_{1}=1.147 \times$ $10^{-3} ; b_{2}=7.82$. Recall respective units. Fig. 3 illustrates development of the above strains during shearing, for the data assumed.

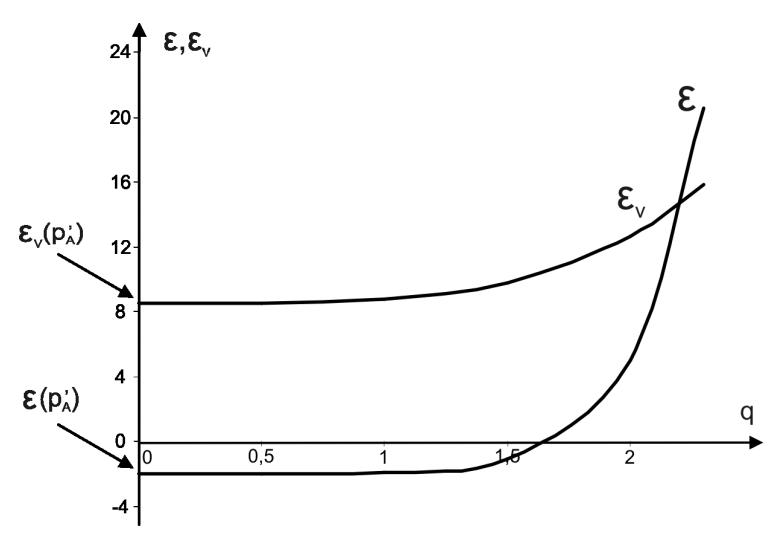

Fig. 3. Volumetric and deviatoric strains that develop during shearing of initially contractive sand 


\section{Initially dilative sand}

In this case, only function $N$ has a different shape, and instead of Eq. (34) we can write the following incremental equation for volumetric strains:

$$
d \varepsilon_{v}=N d q
$$

where

$$
\begin{gathered}
N=\frac{1}{\sqrt{p^{\prime}}}\left(2 a_{1} \eta+a_{2}\right) \text { for } 0<\eta<\eta^{\prime}, \\
N=\frac{1}{\sqrt{p^{\prime}}}\left[a_{3}+a_{5}\left(a_{3} \eta+a_{4}\right)\right] \exp \left(a_{5} \eta\right) \text { for } \eta^{\prime}<\eta<\eta^{\prime \prime},
\end{gathered}
$$

where $\eta^{\prime}$ defines the instability line and $\eta^{\prime \prime}$ the Coulomb-Mohr yield condition.

Integration of this equation gives:

$$
\begin{gathered}
\varepsilon_{v}=\sqrt{p^{\prime}}\left(a_{1} \eta^{2}+a_{2} \eta\right) \quad \text { for } 0<\eta<\eta^{\prime}, \\
\varepsilon_{v}=\sqrt{p^{\prime}}\left(a_{3} \eta+a_{4}\right) \exp \left(a_{5} \eta\right) \text { for } \eta^{\prime}<\eta<\eta^{\prime \prime} .
\end{gathered}
$$

The values of example parameters, corresponding to "Skarpa" sand, are the following: $a_{1}=-1 ; a_{2}=2 ; a_{3}=-0.0106 ; a_{4}=0.0123 ; a_{5}=6.4 ; \eta^{\prime}=1$. Fig. 4 illustrates the development of volumetric strains during shearing of initially dilative "Skarpa" sand in 2D conditions. The character of deviatoric strains is similar to that described before.

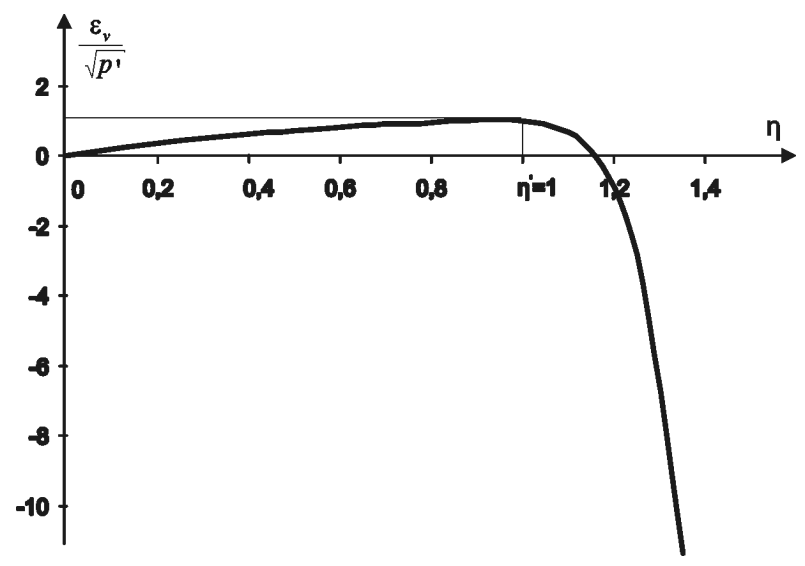

Fig. 4. Development of volumetric strains during shearing of initially dilative "Skarpa" sand 


\subsection{Anisotropic Consolidation}

By anisotropic consolidation we understand the loading along stress path $0 \mathrm{C}$ in Fig. 1 , characterized by $\eta=q / p^{\prime}=$ const. In this case, the incremental equations take the following form:

$$
\begin{gathered}
d \varepsilon_{v}=\frac{\zeta_{v}}{\sqrt{p^{\prime}}} d p^{\prime}, \\
d \varepsilon=\frac{\zeta_{q}}{\sqrt{p^{\prime}}} d p^{\prime},
\end{gathered}
$$

where

$$
\zeta_{v}=\frac{A_{v}}{2}+4 c_{1} \eta^{4} ; \zeta_{q}=\frac{3 A_{q}}{4}+\sqrt{3} \eta b_{1} b_{2} \exp \left(b_{2} \eta\right) .
$$

Integration of Eqs. (40) and (41), assuming zero initial conditions, leads to the following expressions for strains:

$$
\begin{gathered}
\varepsilon_{v}=2 \zeta_{v} \sqrt{p^{\prime}}, \\
\varepsilon=2 \zeta_{q} \sqrt{p^{\prime}} .
\end{gathered}
$$

For initially contractive sand, characterized by parameters presented in previous sections, and assuming $\eta=0.5$, one obtains: $\varepsilon_{v}=7.485 \sqrt{p^{\prime}} ; \varepsilon=-1.41 \sqrt{p^{\prime}}$. Recall respective units.

\subsection{General Algorithm for Integration of Incremental Equations}

The above examples show how to integrate the proposed constitutive equations for the simple loading paths presented in Fig. 1. In these cases analytical formulae for strains have been obtained. In a general case, it is not possible to apply such an easy technique, so some more general comments are necessary. Consider, for example, the stress path shown in Fig. 5. At the beginning, we follow the stress path 0a which is similar to the previously considered case of anisotropic consolidation. Along these paths Eqs. (42) and (43) are valid. Then, we follow path ab, along which there is a deviatoric loading and spherical unloading. Therefore, in constitutive equations (5) and (10) we must choose functions $A$ and $C$ for unloading, and functions $B$ and $D$ for loading. Finally, we follow stress path bc. In this case, we deal with both spherical and deviatoric unloading, so respective functions should be chosen.

In a case of nonlinear loading paths, we must check for each stress increment whether the loading or unloading we deal with is spherical or deviatoric, and then apply respective equations. The proposed approach is certainly different from classical elasto-plastic definitions of loading and unloading. It has been examined in detail in the case of triaxial tests, and it seems that such a definition is physically sound. 


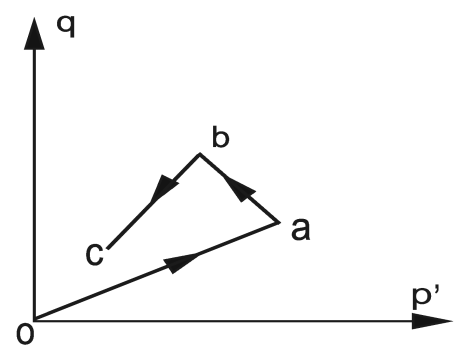

Fig. 5. Complex stress path

\section{Spherical Unloading}

One of the most interesting experiments, performed in triaxial apparatus, deals with spherical unloading of initially contractive sand. The basic idea of this experiment is shown in Fig. 6. First, we consolidate the soil sample by isotropic compression (path $0 \mathrm{~A})$. Then, the stress deviator is applied at constant mean stress (path AB). After that, the deviatoric stress $q$ is kept constant and the mean effective stress $p^{\prime}$ is gradually reduced (spherical unloading). It is interesting to see how the volumetric deformations of the soil sample will develop in this case.

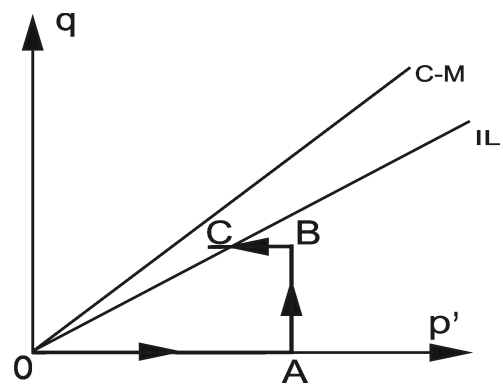

Fig. 6. Stress path corresponding to spherical unloading (BC)

It was shown, in the case of triaxial tests, that spherical unloading exhibits a role of the instability line. At the beginning of unloading, the sample dilates, but after reaching the instability line it suddenly compacts. In the $2 \mathrm{D}$ case considered in this paper, we shall follow a similar procedure. Consider the volumetric changes caused by spherical unloading (path BC). In this case $d q=0$, but $d \eta>0$, so we deal with deviatoric loading. Because $d p^{\prime}<0$ there is a spherical unloading. The respective constitutive equation for the volumetric changes takes the following form:

$$
d \varepsilon_{v}=\frac{1}{\sqrt{p^{\prime}}} F d p^{\prime}
$$


where $F=A_{v}^{u} / 2-4 c_{1} q^{4} /\left(p^{\prime}\right)^{4}$.

Integration of Eq. (44) gives the following formula:

$$
\varepsilon_{v}=\varepsilon_{v}\left(p_{A}, q_{B}\right)+A_{v}^{u}\left(\sqrt{p}-\sqrt{p_{A}}\right)+\frac{8}{7} c_{1} q^{4}\left(p^{-\frac{7}{2}}-p_{A}^{-\frac{7}{2}}\right) .
$$

Fig. 7 illustrates Eq. (45) for the data corresponding to the initially contractive sand.

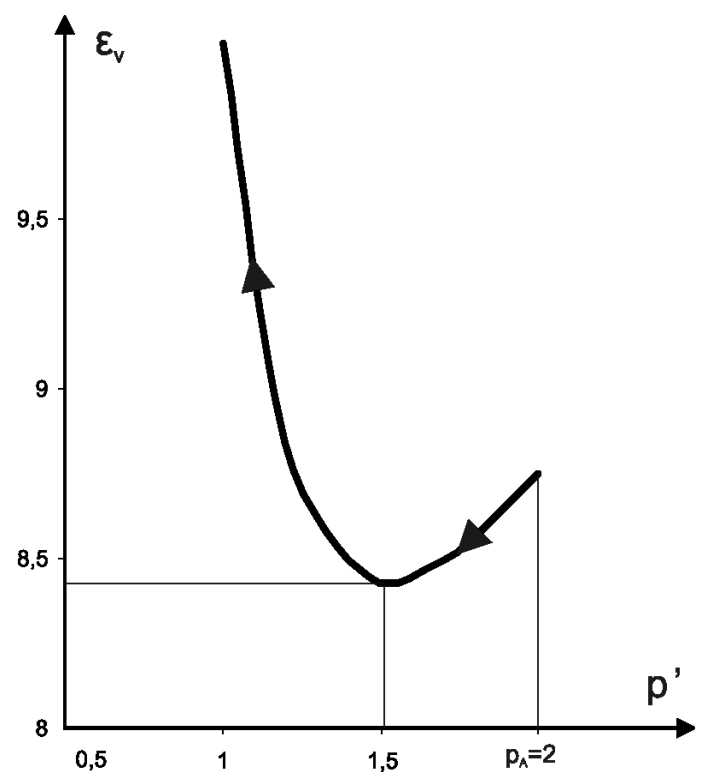

Fig. 7. Volumetric changes during spherical unloading of initially contractive sand

\section{Static Liquefaction}

Another interesting example is shearing of saturated sand in undrained conditions. This problem can also be studied using elasto-plastic models, see Boukpeti et al (2002). For initially contractive sand this leads to static liquefaction. In this case the volumetric changes are given by the following formula:

$$
d \varepsilon_{v}=\frac{A_{v}^{u}}{2 \sqrt{p^{\prime}}} d p^{\prime}+4 \sqrt{p^{\prime}} c_{1} \eta^{3} d \eta=0
$$

It would be convenient in this case to replace the variable $\eta$ with $q$ and re-write Eq. (46), which would take the following form:

$$
\frac{d q}{d p^{\prime}}=H
$$

where

$$
H=\left[4 \sqrt{p^{\prime}} c_{1} \frac{q^{4}}{\left(p^{\prime}\right)^{5}}-\frac{A_{v}^{u}}{2 \sqrt{p^{\prime}}}\right] \times\left[4 \sqrt{p^{\prime}} c_{1} \frac{q^{3}}{\left(p^{\prime}\right)^{4}}\right]^{-1} .
$$


Eq. (47) has been integrated analytically for initially contractive sand and for the presented values of the material's coefficients. The final result is the following:

$$
q=p \times\left[\frac{A_{v}^{u}}{2 c_{1}} \ln \left(\frac{p_{0}}{p}\right)\right]^{\frac{1}{4}} .
$$

Fig. 8 illustrates Eq. (48) for two sets of parameters. The maximum shear stress corresponds to the instability line.

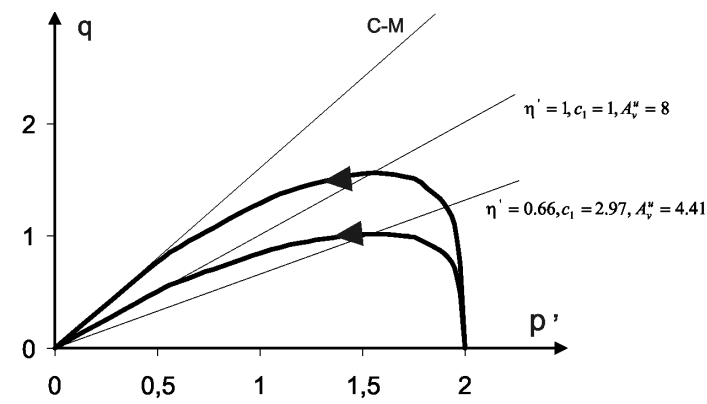

Fig. 8. Effective stress path followed during undrained shearing of initially contractive sand static liquefaction

\section{Instability and Analytical Derivation of Instability Line}

The behaviour of sand analysed in Sections 7 and 8 may be considered as unstable. In the case of spherical unloading, the situation is similar to that described by a simple example. Let us imagine that we squeeze a ball and then reduce this pressure. It is obvious that far we release this pressure the volume of the ball will increase, as during the first stage of spherical unloading, before reaching the instability line in the effective stress space. In our experiment, the ball will take a spherical initial shape when we unload it completely. But initially contractive soil behaves in a different manner. After reaching some threshold (instability line) the volume of soil decreases suddenly, in contrast to the expected behaviour of a ball. It looks as though a hole has been punctured in our ball.

The second example of static liquefaction deals with behaviour of saturated sand in undrained conditions. Such behaviour is typical only for granular media. When we shear such a soil, the effective mean pressure is reduced due to the generation of pore pressure, and finally a saturated sand, which had displayed the properties typical for a solid before liquefaction, behaves like a liquid. This is also a kind of instability.

In this Section, we would like to analyse these problems using well established methods, including Hill's stability condition. This criterion was originally proposed for solid media, and there was no discussion about definition of stresses. In the case of saturated granular soils, a certain distinction is necessary, as we deal with total stresses 
as well as effective stresses. It was shown (Sawicki and Świdziński, 2010c) that Hill's criterion is valid for a dry soil or a soil saturated in undrained conditions. In the case of partly drained soils it leads to ambiguous results. We have decided that a proper formulation of Hill's criterion of stability should be formulated in terms of effective stresses, as it is they are really responsible for the behaviour of soils. Therefore, using this criterion, we would like to analyse the unstable behaviour of sands.

The Hill's second order work has the following form:

$$
d^{2} W=d \sigma_{1} d \varepsilon_{1}+d \sigma_{3}^{d} \varepsilon_{3}=d p^{\prime} d \varepsilon_{v}+\frac{1}{\sqrt{3}} d q d \varepsilon,
$$

The behaviour of material is stable if $d^{2} W>0$. We shall apply this criterion to the behaviour of sand analysed in this paper. In the case of spherical unloading, there is $d q=0$ and the stability criterion requires that:

$$
d^{2} W=d \varepsilon_{v} d p^{\prime}=\frac{1}{\sqrt{p^{\prime}}} F\left(d p^{\prime}\right)^{2}>0 .
$$

This condition means that there should be $F>0$, which leads to the following stability criterion:

$$
\eta^{4}<\frac{A_{v}^{u}}{8 c_{1}}=\left(\eta^{\prime}\right)^{4}
$$

where $\eta^{\prime}$ denotes the instability line. This means physically that the sand behaviour before reaching the instability line is similar to above described our ball example. For $\eta>\eta^{\prime}$ this behaviour becomes unstable.

In the case of undrained shearing, leading to static liquefaction, the second order work is given by the following formula:

$$
d^{2} W=\frac{2}{\sqrt{3}} d q d \varepsilon_{1}
$$

as $\varepsilon_{3}=-\varepsilon_{1}$ in undrained conditions. Some simple manipulations lead to the following expression:

$$
d^{2} W=\frac{2}{\sqrt{3}} H d p^{\prime} d \varepsilon_{1} .
$$

In the case considered, there is always $d \varepsilon_{1}>0 ; d p^{\prime}<0$ therefore, for stable behaviour the following condition should be satisfied: $H<0$. This condition leads to the following criterion for stable behaviour:

$$
\eta<\eta^{\prime}
$$

which is again consistent with experimental data, as after reaching the instability line in the effective stress space, the process of static liquefaction is triggered.

Both conditions $F=0$ and $H=0$ lead to the same formula for the instability line:

$$
\eta^{\prime}=\left(\frac{A_{v}^{u}}{8 c_{1}}\right)^{1 / 4} \text {. }
$$


This formula differs slightly from that obtained for the triaxial case, where instead of 8 in the denominator of Eq. (55) we had 7. But note that we have considered different configurations. An interesting feature of the above derivation is that we have obtained analytically the same formula for the instability line for two different cases. It is also interesting that the instability line has emerged for the behaviour of initially contractive soils, without any presumption.

\section{Discussion and Conclusions}

1. The original 2D model describing pre-failure behaviour of sand is presented. The model takes into account such features as initial anisotropy, initial state defined as contractive or dilative and applies for both dry/fully drained sands and undrained conditions. It describes pre-failure deformations of sand and such phenomena as static liquefaction. An original definition of loading/unloading has been proposed, which does not need a notion of yield surface. Incremental equations describing the model have a rather simple shape, and they can easily be integrated for more complex loading paths. The respective algorithm was presented.

2. The model also takes into account pre-failure instabilities of sand, both drained and undrained. A modified Hill's condition has been applied to study these instabilities. An original Hill's criterion was formulated in terms of total stresses. We have introduced effective stresses into this condition. Sawicki and Świdziński (2010c) have shown that this does not matter for fully drained and undrained conditions, but in the case of partial drainage such a distinction is important. The applied approach has allowed for analytical derivation of the instability line, which is an important achievement.

3. The model was calibrated indirectly, using experimental data from triaxial experiments. Respective constitutive functions have been transformed from triaxial to plane strain conditions using the methods of tensor algebra. The respective procedure for calibration of the model in the plane strain apparatus was described. As previously mentioned, we had no access to the real plane state apparatus, so a direct calibration of our model was not possible. Available experimental data, found in such other publications as, for example, Chu and Wanatowski (2009) are not sufficient to calibrate our model. However, it is possible to perform experiments according to suggestions presented in this paper in order to calibrate the model directly and to validate it for stress paths different from those used for calibration. Such an exercise would show whether our method of calibration, based on triaxial tests, is sufficient or not. We have presented some predictions of our model for the plane strain conditions. These predictions can be checked in laboratories which have access to plane strain apparatuses.

4. The model proposed is a simple alternative to complicated elasto-plastic or hypoplastic approaches. It is based on bare experimental data. In the case of triaxial 
tests, the model's predictions were very good. It is hoped that it also will work well for the plane strain conditions.

5. Obviously, the model proposed has some limitations. It was checked for monotonic loadings. In the case of cyclic loadings, this model is probably impractical, as it requires integration of incremental equations over a large number of cycles. The model is also restricted to "compression tests", as we have had not sufficient data from "extension tests". This means that we have not introduced the third invariant of the stress tensor into our considerations.

\section{APPENDIX \\ Basic differences between the Elasto-Plastic Modelling (EP) of granular soils and Empirical Incremental Equations (EIEP) presented in this paper}

The approach presented in this paper, as well as in the previous authors' publications, differs significantly from basic assumptions of elasto-plasticity. Elasto-plasticity has had a long history and most of geotechnical authors have been anchored to this paradigm. For this reason, the EIE modelling has often been criticized, so it is necessary to list the basic differences between these two approaches and repeat arguments supporting the EIE idea. Note, that we do not reject the EP modelling, but only propose a kind of its supplement, and perhaps some alternative.

\section{States of Soil}

In the theory of EP, two states of the soil are postulated, namely the elastic and plastic. In order to distinguish these two states, a certain function, designated as the yield condition $(f=0)$ is introduced. The stress states defined by $f<0$ correspond to the elastic behaviour of soil, whilst the plastic behaviour is possible only when $f=0$. The yield condition can be geometrically represented as a surface in the stress space, as schematically shown in Fig. A1.

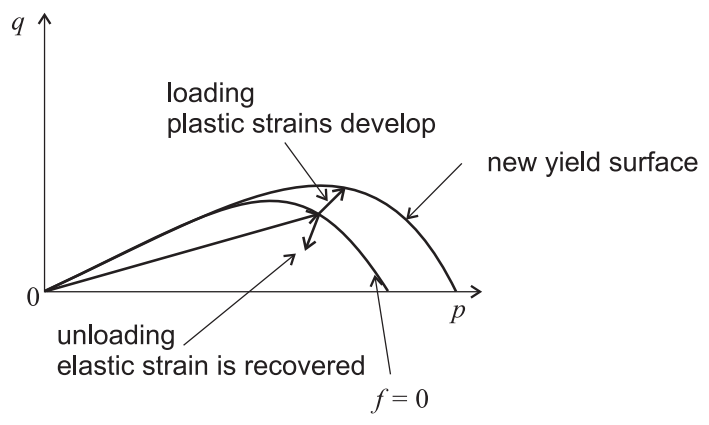

Fig. A1. Illustration of basic concepts of elasto-plasticity

We have used traditional symbols, where $q=$ stress deviator and $p=$ mean stress. For the sake of simplicity, we are considering the fully drained soil, which means 
that $p$ equals to the mean effective stress. The states lying inside the yield surface correspond to the elastic behaviour of soil. The plastic strains can develop only when the stress state satisfies the condition $f=0$.

In the case of EIE, we do not introduce a yield surface, except for the limit/critical state surface (Coulomb-Mohr), which corresponds to the ultimate state of soil. This means that we do not distinguish between the elastic and plastic strains inside the limit surface, as we deal with the total strains. Various experimental results show that the plastic (irreversible) strains develop in soils almost from the very beginning of loading. It is very difficult to determine the elastic range of soil behaviour. The concept of current yield surface has only a theoretical meaning, as we still do not have sound empirical arguments supporting this idea. In the EIE approach this idea is not introduced.

\section{Definition of Loading and Unloading}

This is a key issue in modelling the pre-failure behaviour of soils. In the EP approach, this problem is solved by introducing the concept of yield surface. The loading is defined by the following relations: $f=0, d f \geq 0$. This corresponds to the stress increments tangent to a current yield surface or directed outwards (see Fig. A1). Only during the loading, the plastic strains can develop. They can be determined from independemt flow rule, which should be defined for particular model. Unloading is defined by the relations: $f=0, d f<0$, which correspond to the stress increments directed inwards the current yield surface Fig. A1.

The above approach means that the definition of loading/unloading depends on the shape of yield surface, which is different in various approaches. A consequence of such a definition is that the same stress increment may correspond to loading according to one theory, and unloading according to the other model. Such an approach is not acceptable, as the plastic strains should not depend on the definition of yield surface, as they are objective from the physical point of view.

In the EIE approach, we avoid such an ambigous situation, an objective definition of the loading and unloading is introduced, which depends only on the direction of subsequent stress increments. We distinguish the spherical and deviatoric loading/unloading, as shown in Fig. A2.

The stress increments, from point $\mathrm{A}$ in the stress space, may be directed into four different regions. Each of possible choices involves different loading/unloading criteria, as illustrated in Fig. A2. According to these criteria, we choose respective functions in the constitutive equations, which are defined uniquely, for the loading and unloading respectively. Consider a general shape of constitutive equations:

$$
\begin{gathered}
d \varepsilon_{v}=M d p+N d q, \\
d \varepsilon_{q}=P d p+Q d q .
\end{gathered}
$$




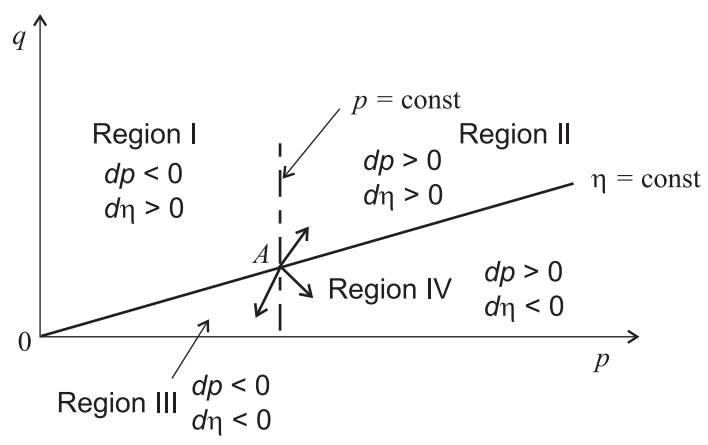

Fig. A2. Loading and unloading in the EIE approach

These equations can also be presented, in the alternative form, using the non-dimensional stress $\eta=q / p$ :

$$
\begin{aligned}
& d \varepsilon_{v}=(M+\eta N) d p+N p d \eta, \\
& d \varepsilon_{q}=(P+\eta Q) d p+Q p d \eta .
\end{aligned}
$$

The functions $M, N, P, Q$ have unique meaning for loading and unloading. There is continuity of strain for each stress increment, and Eqs. (A1)-(A4) can be integrated along arbitrary stress path. The lines $p=$ const $d p=0$ and $\eta=$ const $d \eta=0$ have a particular meaning as they divide respective regions I-IV. Integration of incremental equations along these lines is straightforward. For example, if the stress path coincides with the line $\eta=$ const, respective strain increments can be obtained from Eqs. (A3) and (A4) by putting $d \eta=0$. The line $p=$ const corresponds to pure shearing, so respective strain increments can be obtained from Eqs. (A1) and (A2), just by putting $d p=0$. The same result can be obtained from Eqs. (A3) and (A4).

\section{Other differences}

One of the other important differences is that the EIE approach takes into account the initial state of soil, defined either as contractive or dilative. This important distinction has usually been ignored in many of EP models. The EIE model takes also into account the initial anisotropy, which usually is disregarded in most of EP models, although it is experimentally observed. 


\section{References}

Bazant Z. P. and Krizek R. J. (1976) Endochronic constitutive law for liquefaction of sand, Jnl Engineering Mechanics Div., ASCE, 2, 225-238.

Boukpeti N., Mróz Z. and Drescher A. (2002) A model for static liquefaction in triaxial compression and extension, Canadian Geotechnical Jnl, 39 (6), 1243-1253, DOI: 10.1139/ T02-066.

Chu J. and Wanatowski D. (2009) Effect of loading mode on strain softening and instability behavior of sand in plane-strain tests, Jnl Geotechnical and Geoenvironmental Engineering, ASCE, 135 (1), 108-120, DOI: 10.1061/(ASCE)1090-0241(2009)135:(108).

Głębowicz K. (2006) Hypoplastic modelling of pre-failure behaviour of sand against experimental data, Archives of Hydro-Engineering and Environmental Mechanics, 53 (1), 31-47.

Kolymbas D. (2000a) Introduction to Hypoplasticity, Advances in Geotechnical Engineering and Tunneling, Balkema, Rotterdam/Brookfield.

Kolymbas D. (2000b) The misery of constitutive modeling, [in:] Constitutive Modelling of Granular Materials (ed. D. Kolymbas), Springer, Berlin - Heidelberg - New York, 11-24.

Lade P. V. (1992) Static instability and liquefaction of loose fine sandy slopes, Jnl Geotechnical Engineering, ASCE, 118 (1), 51-71.

Lade P. V., Nelson R. B. and Ito Y. M. (1987) Nonassociated flow and stability of granular materials, Jnl Engineering Mechanics, ASCE, 113 (9), 1302-1318.

Mróz Z., Boukpeti N. and Drescher A. (2003) Constitutive model for static liquefaction, Int. Jnl of Geomechanics, ASCE, 3 (2), 133-144, DOI: 10.1061/(ASCE) 1532-3641. (2003)3:2(133)

Saada A. and Bianchini G. (eds) (1987) Constitutive equations for granular non-cohesive soils, Proc. Int. Workshop, Cleveland, Balkema, Rotterdam/Brookfield.

Sawicki A. (2003) Cam-clay approach to modelling pre-failure behaviour of sand against experimental data, Archives of Hydro-Engineering and Environmental Mechanics, 50 (3), 229-249.

Sawicki A. (2008) 3D and 2D formulations of incremental stress-strain relations for granular soils, Archives of Hydro-Engineering and Environmental Mechanics, 55 (1-2), 45-53.

Sawicki A. and Świdziński W. (2010a) Stress-strain relations for dry and saturated sands, Part I: Incremental model, Jnl Theoretical and Applied Mechanics, 48 (2), 309-328.

Sawicki A. and Świdziński W. (2010b) Stress-strain relations for dry and saturated sands, Part II: Predictions, Jnl Theoretical and Applied Mechanics, 48 (2), 329-373.

Sawicki A. and Świdziński W. (2010c) Modelling the pre-failure instabilities of sand, submitted for possible publication, DOI: 10.1016/j.compgeo.2010.06.004.

Sladen J. A., D'Hollander R. D. and Krahn J. (1985) The liquefaction of sands, a collapse surface approach, Canadian Geotechnical Jnl, 22 (4), 564-578.

Valanis K. C. and Read H. E. (1982) A new endochronic plasticity model for soils, [in:] Soil Mechanics - Transient and Cyclic Loads (G. N. Pande and O. C. Zienkiewicz, eds), John Wiley and Sons, $375-417$. 